\title{
Accurate Measurement of Electric Potential Distributions at the Interfaces in Solids Using Phase-shifting Electron Holography
}

Tsukasa Hirayama ${ }^{1}$, Satoshi Anada ${ }^{1}$, Yuki Nomura ${ }^{2}$, Hirokazu Sasaki $^{3}$, Koh Saitoh ${ }^{4}$ and Kazuo Yamamoto ${ }^{1}$

${ }^{1}$ Japan Fine Ceramics Center, Nagoya, Aichi, Japan, ${ }^{2}$ Panasonic Corporation, Moriguchi, Osaka, Japan, ${ }^{3}$ Furukawa Electric Co. Ltd., Yokohama, Kanagawa, Japan, ${ }^{4}$ Nagoya University, Nagoya, Aichi, Japan

When two materials come in contact, a potential step is formed at the interface by means of the redistribution of mobile carriers according to the potential difference. As this potential step often creates useful properties in materials and devices, the accurate measurement of this potential step is vital and valuable to understand the mechanism governing function generation and control these properties.

Electron holography is a powerful method for visualizing electromagnetic fields at nanoscale ranges [1]. In particular, phase-shifting electron holography is a promising technique to measure electric potential distributions accurately [2,3], and we have employed this technique to study semiconductor devices and battery materials. This paper presents two typical applications of phase-shifting electron holography. One is the examination of p-n junctions, and the other is the direct measurement of the space-charge layer formed at the electrode/electrolyte interface.

Figure 1 shows the electric potential step measured across a p-n junction in GaAs. A TEM specimen was carefully prepared by the Focused Ion Beam (FIB) Method and observed by phase-shifting electron holography using an HF-3300EH electron holography microscope [4]. From the relationship between the applied voltage and the measured potential step across the p-n junction, the built-in potential across the p-n junction was determined to be $1.55 \mathrm{~V}$, which agrees well with the theoretically predicted value.

Figure 2 shows the directly observed and measured electric potential distributions in the vicinity of the $\mathrm{Cu} /$ $\mathrm{Li}_{1+\mathrm{x}+\mathrm{y}} \mathrm{Al}_{\mathrm{x}}(\mathrm{Ti}, \mathrm{Ge})_{2-\mathrm{x}} \mathrm{Si}_{\mathrm{y}} \mathrm{P}_{3-\mathrm{y}} \mathrm{O}_{12}$ (LASGTP) interface [5]. After the TEM specimen was prepared by means of FIB milling, the entire surface of the TEM specimen was completely covered with an amorphous $\mathrm{Al}_{2} \mathrm{O}_{3}$ insulating film (20 nm thick) by atomic layer deposition (ALD). Subsequently, a conductive carbon film (10 $\mathrm{nm}$ thick) was deposited on the specimen, and the carbon film was grounded to prevent electric charging due to incident electron beam irradiation. The space charge layer at the interface (potential difference $\sim 1.3 \mathrm{~V}$, thickness $\sim 10 \mathrm{~nm}$ ) was successfully measured directly for the first time.

In conclusion, phase-shifting electron holography enables us to measure electric potentials in solids accurately and is useful not only for scientific studies but also for supporting research and development in the industry [6].
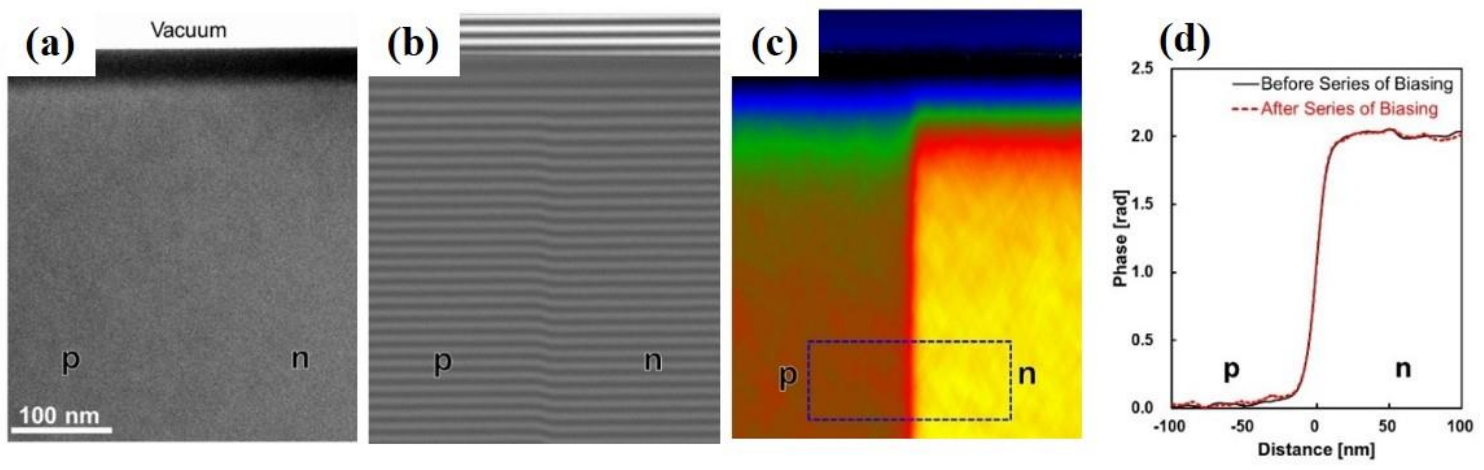
Figure 1. Electric potential step measured at a p-n junction in GaAs. (a) TEM image, (b) hologram, (c) phase image, and (d) phase profile.

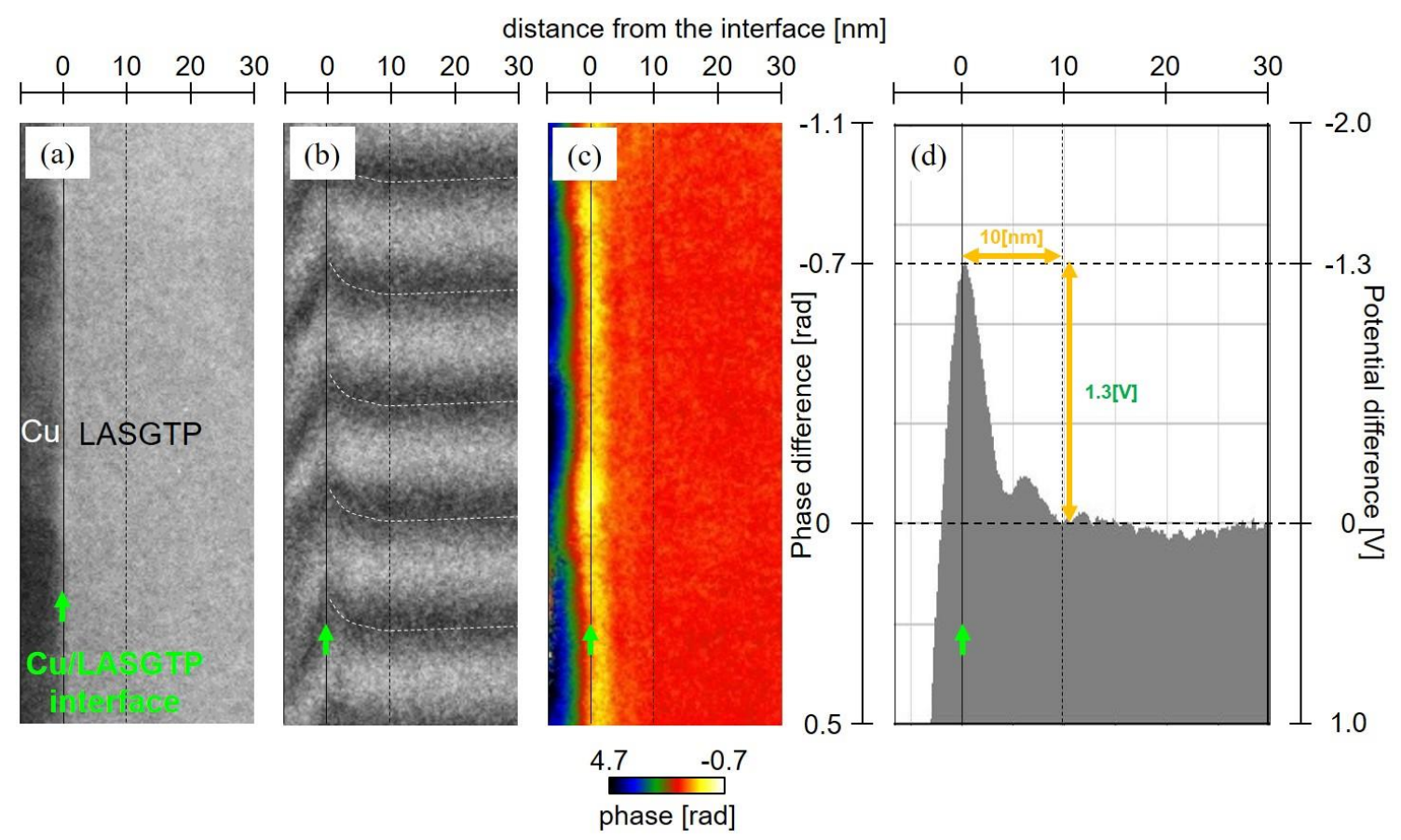

Figure 2. Electric potential distributions measured in the vicinity of the Cu/LASGTP interface using phaseshifting electron holography. (a) TEM image, (b) hologram, (c) phase map, and (d) electric potential profile.

\section{References}

[1] A. Tonomura, Electron Holography 2nd ed. (Springer, New York, 1999).

[2] Q. Ru et al., Appl. Phys. Lett. 59, 2372 (1991).

[3] K. Yamamoto et al., J. Electron Microsc. 49, 31 (2000).

[4] S. Anada et al., Appl. Phys. Lett. 122, 225702 (2017).

[5] Y. Nomura et al., Angew. Chem. Int. Ed. 58, 5292 (2019).

[6] We thank Dr. Yujin Hori, Dr. Kouhei Kinugawa, Dr. Akihiro Imamura of Furukawa Electric Co. Ltd., Dr. Satoru Ouchi, Dr. Emiko Egaki of Panasonic Corporation, and Prof. Naoya Shibata of the University of Tokyo for valuable discussions. This research was partially supported by the Project for Promoting Public Utilization of the Advanced Research Infrastructure Grant from the Ministry of Education, Culture, Sports, Science, and Technology, and Japan Society for the Promotion of Science (JSPS), Grant-in-Aid for Scientific Research KAKENHI (JP 17H02792). 